\title{
TUMOR VENÉREO TRANSMISSÍVEL (TVT): ESTUDO RETROSPECTIVO DE 144 CASOS
}

\author{
VENEREAL TRANSMISSIBLE TUMOR (TVT): RETROSPECTIVE STUDY OF 144 CASES
}

\author{
R. R. HUPPES ${ }^{1}$, C. G. SILVA ${ }^{2}$, R. A. R. USCATEGUI ${ }^{3}$, A. B. DE NARDI ${ }^{4}$, F. W. SOUZA $^{5}$, \\ M. TINUCCI COSTA ${ }^{6}$, R. L. AMORIM ${ }^{7}$, J. M. PAZZINI ${ }^{8}$, J. L. M. FARIA ${ }^{9}$
}

\begin{abstract}
RESUMO
Objetivando analisar a prevalência de TVT em relação ao sexo, raça, idade, localização da lesão e resposta ao tratamento quimioterapêutico foi realizado um estudo retrospectivo de 144 cães atendidos no Hospital Veterinário da Universidade de Uberaba (HVU) durante os anos de 2007 à 2012, diagnosticados com TVT através da realização de exames de cito ou histopatologia. Destes, $59 \%$ eram cães sem raça definida (SRD). Apesar de 16,7\% dos cães avaliados não terem suas idades determinadas, os resultados desta pesquisa revelaram que os pacientes apresentaram idade variando entre 1 e 15 anos sendo a faixa etária entre 2 e 8 anos (40,2\%) a mais afetada. Em relação ao sexo, este trabalho demonstrou que as fêmeas foram mais acometidas que os machos (66,7 e 33,3\% respetivamente). Observou-se que a principal localização da neoplasia foi a vulva $(65,3 \%)$, seguida pelo pênis $(30,6 \%)$, cavidades nasal $(3,5 \%)$ e oral $(0,7 \%)$. Dentre os 54 cães que foram submetidos ao tratamento no hospital veterinário, 39 concluíram o protocolo, sendo que 33 apresentaram citorredução completa utilizando vincristina como agente único; 3 animais se encontravam em tratamento ao final do estudo e nos 3 casos em que houve quimiorresistência, os pacientes se beneficiaram de doxorrubicina.
\end{abstract}

PALAVRAS-CHAVE: cão, doxorrubicina, vincistina.

\section{SUMMARY}

To analyze the prevalence of TVT in relation to sex, breed, age, lesion location, and response to chemotherapeutic treatment, a retrospective study from 2007 to 2012 was conducted with dogs from the Veterinary Hospital of the University of Uberaba (HVU) diagnosed with TVT by cytology or histopathology. Which, 59\% were mongrel dogs (59\%). Although $16.7 \%$ of these dogs had their ages undetermined, the data of this study showed that patients were aged between 1 and 15 years being the range between 2 and 8 years (40.2\%) the most affected. Regarding sexual predisposition, this study shows that females were more affected than males $(66.7,33.3 \%$ respectively). The primary site of the tumor occurrence was the vulva $(65.3 \%)$, followed by the penis $(30.6 \%)$, nasal $(3.5 \%)$ and oral cavities $(0.7 \%)$. Among $54 \mathrm{dogs}$ that were treated at the hospital $39 \mathrm{dogs}$ completed therapeutic protocol, of these 33 dogs had complete cytoreduction using the single-agent vincristine, 3 animals were still receiving treatment at the end of the study and in the 3 chemoresistance cases, these patients were benefited from doxorubicin treatment.

KEY-WORDS: dogs, doxorubicin, Vincristine.

\footnotetext{
${ }^{1}$ Professor Doutor de Técnica Cirúrgica da Faculdade Ingá - Maringá, PR.

${ }^{2}$ Pós-Graduanda em Medicina Veterinária pela Universidade de Uberaba - UNIUB.

${ }^{3}$ Pós-Graduando em Cirurgia Veterinária pela FCAV - UNESP - Campus de Jaboticabal-SP.

${ }^{4}$ Professor Doutor do Departamento de Clínica e Cirurgia Veterinária da FCAV - UNESP - Campus de Jaboticabal-SP.

${ }^{5}$ Pós-Graduando em Cirurgia Veterinária da UFSM, Santa Maria-RS.

${ }^{6}$ Professora Doutora do Departamento de Clínica e Cirurgia Veterinária da FCAV - UNESP - Campus de Jaboticabal-SP.

${ }^{7}$ Departamento de Clínica Médica da FMVZ - UNESP - Campus de Botucatu-SP.

${ }^{8}$ Pós-Graduando em Cirurgia Veterinária pela FCAV - UNESP - Campus de Jaboticabal-SP.

${ }^{9}$ Pós-Graduanda em Medicina Veterinária pela Universidade de Uberaba-MG. *Autor para correspondência: josipazzini@hotmail.com. Av Antonio Ignácio de Araújo, 170, apto 5, Nova Aparecida, Jaboticabal-SP. CEP.14.883-380.
} 


\section{INTRODUÇÃO}

O tumor venéreo transmissível (TVT) é uma neoplasia de células redondas que acomete principalmente cães errantes e sua maior incidência ocorre naqueles sem raça definida. Sua ocorrência é de origem natural e sua transmissão ocorre principalmente através do coito, sendo a vulva, pênis e prepúcio os principais pontos de implantação desta neoplasia (TINUCCI-COSTA, 2009).

Existem outras formas de transmissão do TVT, por exemplo, o hábito de lamber e farejar dos cães leva à implantação de células tumorais na cavidade oral ou nasal. Há também relatos de lesões em olhos e pele, classificadas como primárias ou metastáticas (LAPA, 2009; TINUCCI-COSTA, 2009).

A idade de maior ocorrência desta neoplasia é entre dois e cinco anos, momento em que os cães estão no auge de sua fase reprodutiva (FONSECA, 2009). Quanto à predisposição sexual, estudos apontam maior incidência em fêmeas comparada aos machos (ALABANESE et al., 2002; BRANDÃO et al., 2002).

Os sinais clínicos podem variar dependendo da localização. $\mathrm{Na}$ vulva, os pacientes apresentam aumento de volume, odor fétido e secreção serosanguinolenta. Em machos, quando localizado em pênis ou prepúcio, podem ser observados aumento de volume, dificuldade de expor o pênis, descarga prepucial serosanguinolenta, odor forte, fimose ou parafimose, hematúria e disúria. Em cavidade nasal pode ocorrer aumento de volume, desconforto respiratório, epistaxe, secreção purulenta ou serosanguinolenta. Na cavidade oral é mais comum a ocorrência de ulceração, dificuldade de mastigação e fístula oronasal. Em pele, as lesões se apresentam como formações nodulares algumas vezes localizadas ou disseminadas, podendo apresentar ulceração ou não (TINUCCI-COSTA， 2009; MORGAN, 2010). O diagnóstico é estabelecido com o auxílio do exame cito ou histopatológico, e raramente se faz necessário a utilização da técnica de imuno-histoquímica (BATISTA et al., 2007).

O tratamento indicado para o TVT é a quimioterapia antineoplásica utilizando como fármaco a vincristina. Outros antineoplásicos também são recomendados comodoxorrubicina, ciclofosfamida e bleomicina, todavia outras modalidades terapêuticas têm sido citadas como, a cirurgia, eletroquimioterapia, terapia fotodinâmica, radioterapia, radiofrequência (RODASKI \& DE NARDI, 2006; LAPA, 2009; TINUCCI-COSTA, 2009; HUPPES et al., 2010)

Com este trabalho objetivou-se analisar a prevalência de TVT em relação ao sexo, raça, idade, localização da lesão, assim como os sinais clínicos e resposta ao tratamento quimioterapêutico.

\section{MATERIAL E MÉTODOS}

Foi realizado um estudo retrospectivo entre os anos de 2007 à 2012 com cães atendidos no Hospital Veterinário da Universidade de Uberaba (HVU), diagnosticados com TVT através da realização de exames cito ou histopatológico. Neste estudo foi realizado o levantamento da idade, raça, sexo, localização do tumor, sinais clínicos e o tratamento instituído. Foi utilizada no tratamento a vincristina como terapia inicial e nos casos em que os cães apresentaram quimo resistência a vincristina foi substituído pela doxorrubicina na tentativa de promover remissão completa da lesão.

Este estudo foi realizado com base na análise das prontuários e os dados dos pacientes avaliados por estatística descritiva.

\section{RESULTADOS E DISCUSSAO}

Ao todo, 144 pacientes receberam diagnóstico de TVT durante o período de avaliação. Destes, 59\% eram cães sem raça definida (SRD) (Figura 1). Como descrito por SILVA et al., (2007) esta observação está relacionada com as condições socioeconômicas das famílias de baixa renda, que não adquirem cães de alto valor zootécnico e permitem o acesso livre de seus animais à rua por não terem um local adequado para a manutenção dos mesmos, propiciando o contato de seus animais de estimação com os cães de rua que geralmente lhes transmitem a doença (HASLER \& WEBER, 2000; PEREIRA et al., 2000; PAPAZOGLOU et al., 2001).

Contudo, esta neoplasia pode ocorrer em cães de raça $(41 \%)$ (Figura 1), mas para que isso ocorra é necessário o contato direto com animais que apresentam a doença, o que é raro entre cães de alto valor zootécnico. Os animais de raça, em sua maioria, são de proprietários que se responsabilizam por seus cães. Estes animais recebem vacinação e vermifugação regularmente, além de terem as condições de habitação necessárias para serem acomodados. Este perfil de proprietário usualmente realiza procedimentos de esterilização precocemente. Todos estes fatores contribuem para redução do risco de transmissão do TVT entre cães de raça, justificando a menor incidência desta neoplasia nestes animais (HASLER \& WEBER, 2000; PEREIRA et al., 2000; PAPAZOGLOU et al., 2001), como também foi comprovado neste estudo.

Dentre os pacientes avaliados, constatou-se que os proprietários não souberam informar a idade de seus cães em $24(16,7 \%)$ dos 144 animais (Figura 2). Isso pode ser justificado pelo fato desta neoplasia ser muito frequente em animais errantes que são adotados (LAPA, 2009; TINUCCI-COSTA, 2009), tornando difícil a determinação precisa da idade destes animais. Por outro lado, mesmo aqueles cães de proprietários, muitos não conseguem informar com exatidão a idade dos seus animais por não acompanharem seu desenvolvimento desde filhotes.

Dos animais que foi obtida a informação a respeito da faixa etária, esta variava entre 1 e 15 anos, sendo que $12,5 \%$ tinham oito anos, $11,8 \%$, sete, $8,3 \%$, cinco e 7,6\%) dois (Figura 2). Resultados semelhantes a este estudo foram relatados por SILVA et al., (2007) que demonstraram maior incidência de TVT em cães de três a cinco anos $68 \%$, seguidos por animais com 
dois anos $(87 \%)$ e seis a oito anos $(12,5 \%)$. Reforçando estas informações, TINUCCI-COSTA (2009) relatam que esta neoplasia acomete com maior frequência animais com idade entre oito meses a sete anos, ou seja, desde o início da idade reprodutiva.

Em relação ao sexo, este trabalho demonstra que as fêmeas são mais acometidas que os machos (66,7 e 33,3\% respetivamente), semelhante ao observado por BRANDÃO et al., (2002). Contudo, TINUCCI-COSTA (2009) afirma que não há predileção sexual entre a população canina acometida por TVT. E segundo SILVA et al. (2007), encontraram uma incidência muito próxima sendo 35 machos e 34 fêmeas em seu estudo.
O maior número de fêmeas observado no presente estudo pode ser explicado pela receptividade da fêmea a vários machos durante seu período de cio e, embora incomum, uma fêmea pode permanecer neste período até por até 30 dias, aumentando a possibilidade de contato com machos portadores de TVT (FELDMAN \& NELSON, 2004).

Observou-se que a localização na qual ocorre mais comumente a neoplasia foi a vulva $(65,3 \%)$, seguido pelo pênis $(30,6 \%)$, cavidades nasal $(3,5 \%)$ e oral $(0,7 \%)$ (Figura 3). A maior incidência de lesões em vulva e pênis é explicada pelo fato deste tumor ser transmitido, principalmente durante o coito (TINUCCICOSTA 2009, LAPA, 2009).

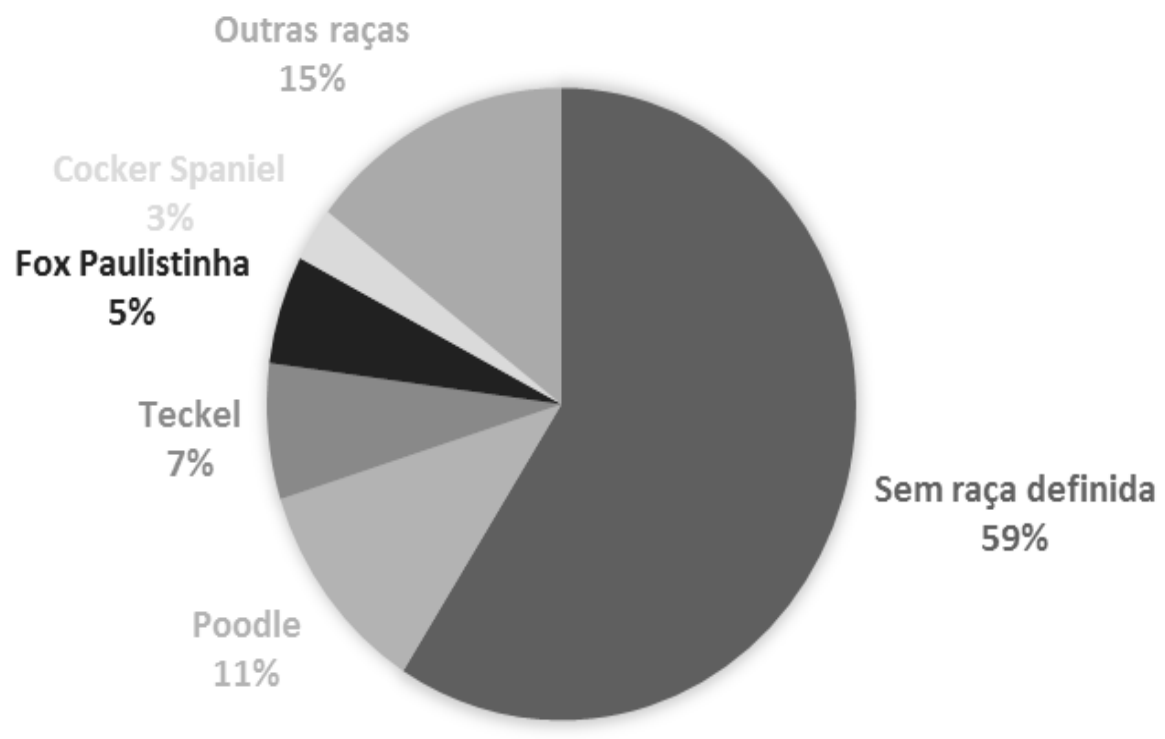

Figura 1 - Representação gráfica da distribuição racial em 144 cães com tumor venéreo transmissível atendidos no Hospital Veterinário da Universidade de Uberaba entre 2007 e 2012.

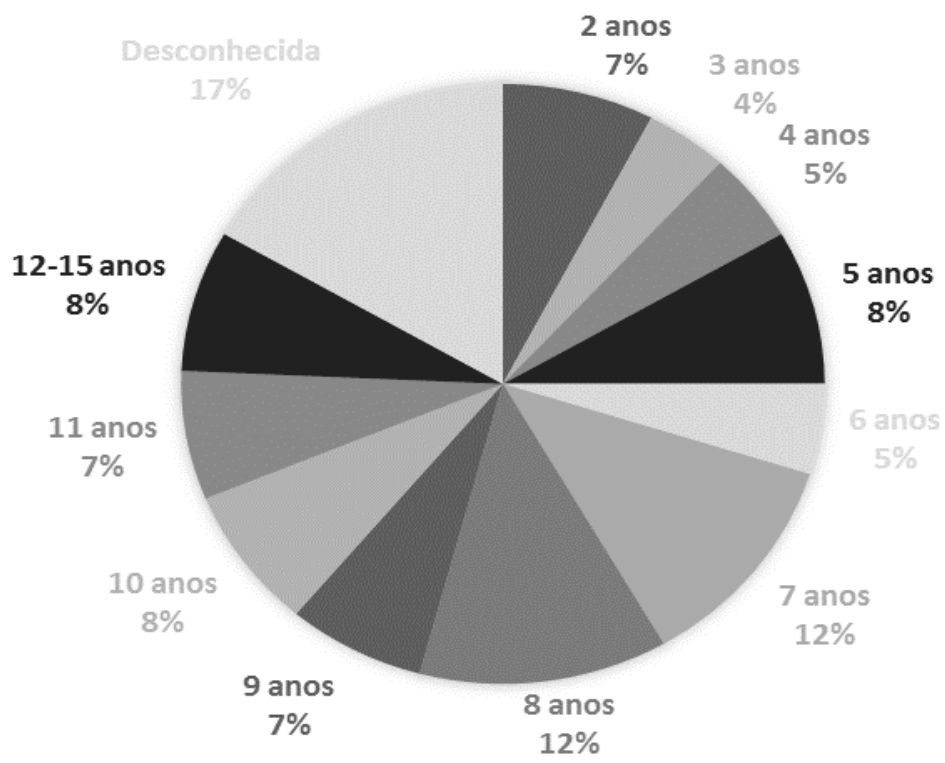

Figura 2 - Representação gráfica da distribuição etária em 144 cães com tumor venéreo transmissível atendidos no Hospital Veterinário da Universidade de Uberaba entre 2007 e 2012. 


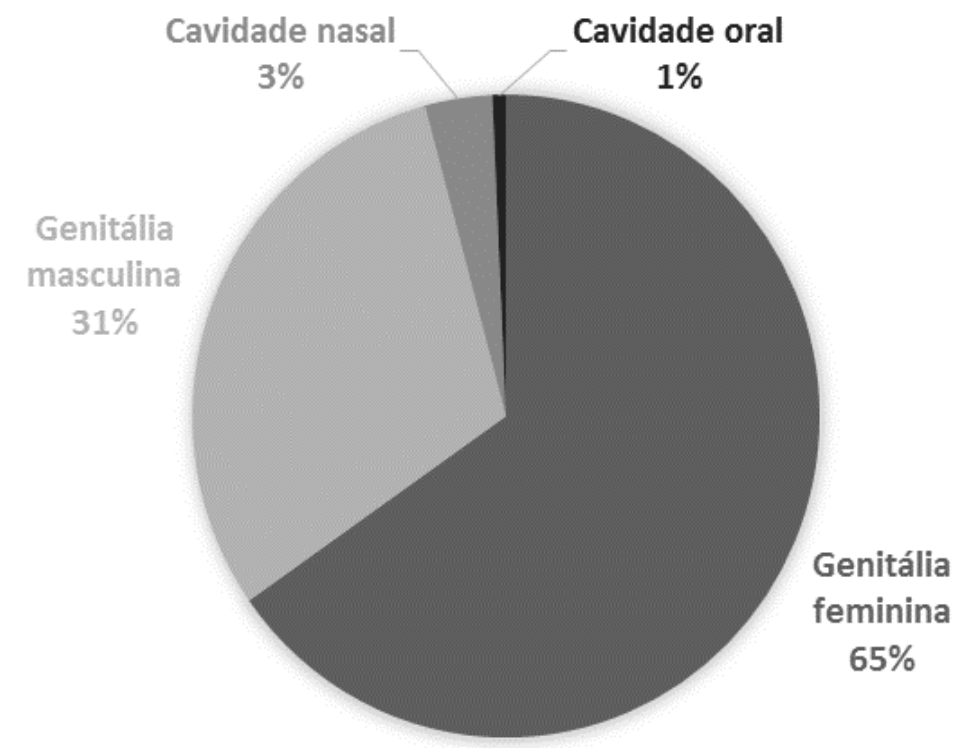

Figura 3 - Representação gráfica da distribuição anatômica das lesões em 144 cães com tumor venéreo transmissível atendidos no Hospital Veterinário da Universidade de Uberaba entre 2007 e 2012.

Tabela 1 - Sessões de Tratamento efetuadas após autorização pelos proprietários em 54 cães com tumor venéreo transmissível no Hospital Veterinário da Universidade de Uberaba entre 2007 e 2012.

\begin{tabular}{cccccc}
\hline Sessão & Tratamento & Animais & Abandono & \multicolumn{2}{c}{ Citorredução } \\
& & & Parcial & 0 \\
\hline 1 & Vincristina & 54 & 4 & 10 & 0 \\
2 & Vincristina & 49 & 6 & 30 & 0 \\
3 & Vincristina & 42 & 5 & 14 & 19 \\
4 & Vincristina & 36 & 0 & 5 & 10 \\
5 & Vincristina & 17 & 0 & 2 & 4 \\
6 & Vincristina & 7 & 0 & 3 & 0 \\
7 & Vincristina / Doxorrubicina & $2 / 1$ & 0 & 2 & 1 \\
9 & Doxurrubicina & 3 & 0 & 0 & 2 \\
\end{tabular}

Tratamento: Vincristina na dose de $0,75 \mathrm{mg} / \mathrm{m}^{2} \mathrm{IV}$, a cada 7 dias, Doxorrubicina na dose de $30 \mathrm{mg} / \mathrm{m}^{2}$, a cada 21 dias. Citorredução avaliada pelo exame histopatológico.

Os sinais clínicos encontrados nos animais que apresentavam lesões genitais (95\%) foram: aumento de volume na genitália, externalização da lesão, secreção serosanguinolenta, deformidade da parede vulvar, dificuldade de exposição peniana, lesões friáveis no bulbo. Estes sinais clínicos são similares aos citados em machos e fêmeas pela literatura (FLORES et al., 1993; LAPA, 2009; TINUCCI-COSTA, 2009).

Observou-se também a ocorrência de TVT em regiões extragenitais, como referenciado por outros pesquisadores (SILVA et al., 2007; LAPA, 2009). Sabe-se que além da genitália externa, o TVT pode ser diagnosticado em outras diversas localizações, tanto em machos, como nas fêmeas (TINUCCI-COSTA,
2009). Em um dos cães deste estudo, a neoplasia foi detectada em cavidade oral apresentando fistula oronasal, ulceração, dificuldade de alimentação e halitose sinais clínicos descritos pela literatura (PAPAZOGLOU et al., 2001; TINUCCI-COSTA, 2009; LAPA 2009).

Em cavidade nasal, as lesões podem causar dificuldade respiratória, deformidade da região plano nasal (aumento de volume), presença de secreção serosanguinolenta ou somente purulenta e epistaxe (PAPAZOGLOU et al., 2001; TINUCCI-COSTA, 2009; LAPA 2009). Dentre os cães que apresentaram lesões nasais neste estudo $(3,5 \%)$, os sinais clínicos corroboram as afirmações anteriores sendo os mais 
comuns foram secreção serosanguinolenta, espirros, dispnéia inspiratória e aumento do volume do plano nasal e um destes pacientes apresentava extensão do tumor para o palato mole.

O desenvolvimento de lesões extra genitais na maioria das vezes ocorre devido ao ato social dos cães de lamber e farejar, principalmente, os genitais, provocando atrito de tecidos que facilita a implantação de células neoplásicas em novos sítios (ALBANESE et al., 2002; SANTOS et al., 2005; LAPA, 2009).

Dos 144 animais diagnosticados com TVT, 90 não foram tratados no HVU, devido à operacionalização do Serviço de Oncologia, os animais foram encaminhados para o tratamento em clínicas particulares, não retornando ao hospital para reavaliação após o tratamento (Tabela 1). Dentre os 54 cães que foram submetidos ao tratamento, apenas 39 o concluíram ou ainda estavam em tratamento ao final do estudo.

O tratamento de eleição para o TVT é a quimioterapia antineoplásica com a utilização de vincristina, na dose $0,75 \mathrm{mg} / \mathrm{m}^{2}$ por via intravenosa (IV), com intervalo entre as administrações de sete dias, totalizando de quatro até oito sessões (RODASKI \& DE NARDI, 2006; LAPA, 2009; TINUCCICOSTA, 2009). Neste estudo, os animais dos proprietários que aceitaram o tratamento quimioterapêutico foram submetidos a este protocolo (54 cães).

Nem sempre os proprietários são convencidos a realizar o tratamento indicado, devido às crenças equivocadas sobre os efeitos colaterais da quimioterapia pela associação com os efeitos que ocorrem em humanos. Somente $20 \%$ dos cães tratados com vincristina, apresentam efeitos colaterais, como distúrbios gastrointestinais, anorexia, alopecia, hiperpigmentação da pele, e quando ocorrem, são na maioria das vezes facilmente controlados (RODASKI\& DE NARDI, 2006).

Outros fatores implicados na desistência dos proprietários são: a necessidade de levar o paciente a cada sete dias ao médico veterinário, as despesas com exames durante o tratamento e o valor dos quimioterápicos antineoplásicos. Devido a estes fatores relatados acima, dos 54 animais tratados no HVU 15 cães não foram levados ao HVU para tratamento quimioterápico antes da remissão completa da lesão. Destes animais, 4 cães receberam uma única aplicação de vincristina, 6 apenas duas sessões e 5 que apresentavam remissão parcial da lesão após três aplicações não retornaram para tratamento nem avaliação, devido principalmente às despesas relacionadas.

Dos 39 cães que completaram o tratamento, 19 apresentaram remissão completa após quatro sessões, 10, após cinco sessões, 4, após seis sessões e 3 encontravam-se ainda em tratamento (entre a segunda e quarta sessão sem remissão total das lesões) ao final do estudo, utilizando como agente único vincristina. Estes resultados corroboram com dados de TINUCCICOSTA (2009) e SILVA et al. (2007), os quais relatam que na maioria das vezes se fazem necessáras quatro a seis sessões de vincristina para remissão completa das lesões.

Outros fármacos como a doxorrubicina, ciclofosfamida, bleomicina e vimblastina, também podem ser utilizados (RODASKI \& DE NARDI, 2006). Estes fármacos podem ser utilizados em protocolos de monoquimioterapia ou poliquimioterapia (SOUZA et al., 2000; TINUCCI-COSTA, 2009, HUPPES et al., 2010). Contudo, vale ressaltar que o fármaco que apresenta os melhores resultados é a vincristina (TINUCCI-COSTA, 2009), como observado neste estudo, no qual $91 \%$ dos pacientes apresentaram remissão completa das lesões com este tratamento.

Após seis e sete sessões com vincristina, 1 e 2 cães respetivamente (8\%) não apresentaram remissão completa das lesões optando-se por substituir o fármaco pela doxorrubicina, na dose de $30 \mathrm{mg} / \mathrm{m}^{2}, \mathrm{IV}$, a cada 21 dias, promovendo remissão completa da neoplasia em ambos os pacientes com duas sessões deste novo quimioterápico. Este fármaco foi instituído pois estudos já demonstraram que este citostático é de eleição em casos de TVT resistentes à vincristina (RODASKI \& DE NARDI, 2006; HUPPES et al., 2010).

\section{CONCLUSÃO}

O TVT é uma neoplasia que afeta principalmente as fêmeas caninas, aos animais sem raça definida e com idades entre 2 e 8 anos. Suas lesões se localizam geralmente na vulva, vagina, pênis e prepúcio, produzindo sinais clínicos caraterísticos, outros órgãos também são afetados porém em baixa proporção. O tratamento com vincristina na dose de $0,75 \mathrm{mg} / \mathrm{m}^{2} \mathrm{IV}$, com intervalo entre as administrações de sete dias continua sendo muito efetivo e de eleição, principalmente após 4 a 6 sessões de aplicação. Nos casos em que existe resistência após 6 semanas, a doxorrubicina na dose $30 \mathrm{mg} / \mathrm{m}^{2} \mathrm{IV}$, a cada 21 dias, pode ser indicada, esperando resultados positivos nas primeiras duas sessões de aplicação.

\section{REFERÊNCIAS}

ALBANESE, F.; POLI, A.; MILLANTA, F.; ABROMO, F. Primary cutaneous extragenital canine transmissible venereal tumour with Leishamania-laden neoplastic cells: a further suggestion of histiocytic origin? Veterinary Dermatology, v. 13, n. 5, p. 243246, 2002.

BATISTA, J. S.; SOARES, S. H.; PERREIRA, R. H. M. A.; PETRI, A. ; SOUZA, F. D. N.; NUNES, F. C. $\mathrm{R}$. Tumor venéreo transmissível intraocular e metástase no baço. Acta Veterinária Brasílica, v. 1, n. 1, p. 4548, 2007.

BRANDÃO, C. V.; BORGES, A. G.; RANZONI, J. J. T.; RAHAL, S. C.; TEIXEIRA, C. R.; ROCHA, N. S. Tumor venéreo transmissível: estudo retrospectivo de 127 casos (1998-2000). Revista Educação Continuada - CRMV-SP, v. 5, n. 1, p. 25-31, 2002. 
FELDMAN, E. C.; NELSON, R. W. Ovarian cycle and vaginal cytology. In: Canine and Feline Endocrinology and Reproduction. St. Lois, Missouri: Elsevier, 2004. cap. 19, p. 752-774.

FLORES, P. E.; DIEZ, Y. X.; DIAZ, R. A. M.; URCELAY, V. S.; CATTANEO, U. G. Comparison of the neoplasms recorded in two periods (1981-1985 and 1986-1988) at the surgery section of the faculty of Veterinary Medicine, Avances en Ciencias Veterinarias, v. 8, n. 1, p. 61-65, 1993.

FONSECA, L. S. Tumor venéreo transmissível espontâneo canino: A inserção do transposon line-1 no gene C-MYC e os critérios de malignidade. Botucatu: Universidade Estadual Paulista Júlio De Mesquita Filho, , 2009. p. 81. Tese (Mestrado em Veterinária) - Faculdade de Ciências Agrárias e Veterinárias, 2009.

HASLER, A. H.; WEBER, W. T. Theriogenology question of the month. Transmissible venereal tumor (TVT). Journal of American Veterinary Medical Association, v. 216, n. 10, p. 1557-1559, 2000. Errata em: Theriogenology question of the month. Journal of American Veterinary Medical Association, v. 217, n. 1, p. 42,2000

HUPPES, R.R.; DE NARDI. A.B.; RAPOSO, T.M.M.; PASSOS, B.L.S.; DALECK, C.R.; SILVA, A.R.; BRUM, A.M. TAGUCHI, T.M., 2010. Utilização da Bleomicina no tratamento de um TVT resistente a Vincristina e Doxorrubicina. VI ONCOVETSimpósio de oncologia veterinária. Realizado em Águas de Líndoia-SP.

LAPA, F. A. S. Estudo comparativo da eficácia de dois protocolos de tratamento de tumor venéreo transmissível em cães. 2009. p. 73. Dissertação (Mestrado em Ciências Animal-Fisiopatologia Animal) - Universidade do Oeste Paulista, Presidente Prudente.
MORGAN, J. D. S. Chemotherapy administration. In: Cancer Management in Small Animal Practice. Copyright: 2010. cap. 4, p. 101-114.

PAPAZOGLOU, L. G.; KOUTINAS, A. F.; PLEVRAKI, A. G.; TONTIS, D. Primary intranasal transmissible venereal tumour in the dog: a retrospective study of six spontaneous cases. Journal of Veterinary Medical. A, Physiology, pathology, clinical Medicine, v. 48, n. 7, p. 391-400, 2001.

PEREIRA, J. S.; SILVA, A. B.; MARTINS, A. L.; FERREIRA, A. M. Immunohistochemical characterization of intraocular metastasis of a canine transmissible venereal tumour. Veterinary Ophthamology, v. 3, n. 1, p. 43-47, 2000.

RODASKI, S.; DE NARDI, A. B. Classificação dos quimioterápicos. In: RODASKI, S.; DE NARDI, A. B. Quimioterapia Antineoplásica em Cães e Gatos. Curitiba: Bio, 2006. cap. 2, p. 98-134.

SANTOS, F. G. A.; VASCONCELOS, A. C.; NUNES, J. E. S.; CASSALI, G. D.; BIOSCI, J. O tumor venéreo transmissível canino - aspectos gerais e abordagens moleculares (revisão da literatura). Journal of Bioscience, v. 21. p. 41-53, 2005.

SILVA, M.C.V.; BARBOSA, R.R..; SANTOS, R.C.; CHAGAS, S.N.; COSTA, W.P. Avaliação epidemiológica $\mathrm{e}$ terapêutica do tumor venéreo transmissível (TVT) na população canina atendida no hospital veterinário da UFESA. Acta Veterinaria Brasílica, v.1 n.1, p. 28-32, 2007.

TINUCCI-COSTA, M. T. Tumor venéreo transmissível canino. In: DALECK, C. R.; DE NARDI, A. B.; RODASKI, S. Oncologia em Cães e Gatos. São Paulo: Roca, 2009. cap. 34, p. 540-551. 